\title{
Coordinate expression of heme and globin is essential for effective erythropoiesis
}

\author{
Raymond T. Doty, Susan R. Phelps, Christina Shadle, Marilyn Sanchez-Bonilla, Siobán B. Keel, and Janis L. Abkowitz \\ Division of Hematology, Department of Medicine, University of Washington, Seattle, Washington, USA
}

\begin{abstract}
Erythropoiesis requires rapid and extensive hemoglobin production. Heme activates globin transcription and translation; therefore, heme synthesis must precede globin synthesis. As free heme is a potent inducer of oxidative damage, its levels within cellular compartments require stringent regulation. Mice lacking the heme exporter FLVCR1 have a severe macrocytic anemia; however, the mechanisms that underlie erythropoiesis dysfunction in these animals are unclear. Here, we determined that erythropoiesis failure occurs in these animals at the CFU-E/proerythroblast stage, a point at which the transferrin receptor (CD71) is upregulated, iron is imported, and heme is synthesized - before ample globin is produced. From the CFU-E/proerythroblast (CD71+ Ter119- cells) stage onward, erythroid progenitors exhibited excess heme content, increased cytoplasmic ROS, and increased apoptosis. Reducing heme synthesis in FLVCR1-defient animals via genetic and biochemical approaches improved the anemia, implying that heme excess causes, and is not just associated with, the erythroid marrow failure. Expression of the cell surface FLVCR1 isoform, but not the mitochondrial FLVCR1 isoform, restored normal rbc production, demonstrating that cellular heme export is essential. Together, these studies provide insight into how heme is regulated to allow effective erythropoiesis, show that erythropoiesis fails when heme is excessive, and emphasize the importance of evaluating Ter119- erythroid cells when studying erythroid marrow failure in murine models.
\end{abstract}

\section{Introduction}

The synthesis of heme is uniquely regulated in developing erythroblasts. The first, and rate-limiting, enzymatic step is mediated by 5 -aminolevulinate synthase 2 (ALAS2) in erythroblasts and ALAS1 in all other cells. While ALAS1 is transcriptionally, translationally, and posttranslationally regulated by heme (1-3), ALAS2 expression is dependent on adequate iron to relieve IRE/IRPmediated inhibition but lacks this direct feedback inhibition by heme (4-6). Thus, the regulation of heme synthesis via feedback inhibition is active in all tissues except developing erythroblasts wherein iron availability drives heme synthesis without feedback regulation. This differential regulation likely reflects the fact that $95 \%$ of rbc protein content is hemoglobin, and thus developing erythroblasts need to produce high levels of heme. Heme itself is necessary to induce globin transcription (7) and translation (8); therefore, heme production must precede and may exceed the levels of heme utilization in hemoglobin during early erythropoiesis. Excess heme will inhibit transferrin-bound iron utilization in erythroblasts (9) and result in the generation of heme-mediated ROS, leading to cell damage and death (10). Therefore, any excess heme must be detoxified either by degradation or elimination.

Heme degradation via heme oxygenase (HMOX) is the primary mechanism that most cells employ to handle excess heme. While recent evidence suggests HMOX is present and active in late-stage fetal erythroblasts and erythroid cell lines (11), it is unclear whether HMOX is present and functional throughout erythroid cell differentiation postnatally. Furthermore, it is not

Conflict of interest: The authors have declared that no conflict of interest exists. Submitted: July 6, 2015; Accepted: October 8, 2015.

Reference information: J Clin Invest. 2015;125(12):4681-4691. doi:10.1172/JCI83054. known how heme degradation versus utilization are prioritized at the various stages of rbc development. Direct export of heme via FLVCR1 (12) provides an alternative cellular mechanism to handle excess heme when its supply exceeds globin availability.

In this study, we show erythroblasts from mice lacking the heme export protein FLVCR1 (13) have elevated heme, ROS, and apoptosis, and that adult erythropoiesis requires the cell surface isoform of FLVCR1, FLVCR1a, but not the mitochondrial isoform, FLVCR1b (14). In addition, we demonstrate that decreasing heme synthesis in mice with induced deletion of both FLVCR1a and FLVCR1b (Flvcr1-deleted mice) improves their rbc production, which implies that excess heme causes the erythroid marrow failure. These data may provide new pathophysiological insight into why human erythroblasts die in disease states where heme supply likely exceeds its utilization, such as Diamond Blackfan anemia and myelodysplasia $(13,15)$.

\section{Results}

The loss of FLVCR1 results in ineffective erythropoiesis and macrocytic anemia. Flvcr1-deleted mice have a block in erythroid differentiation at the CFU-E/proerythroblast stage, resulting in severe macrocytic anemia (13). Complete blood count (CBC) analysis reveals a $50 \%$ decrease of rbc numbers and hemoglobin levels by 4 weeks after deletion (Figure 1), consistent with a near complete developmental block based on mouse erythrocyte lifespan $(16,17)$. The anemia in Flvcr1-deleted mice stabilizes after 8 weeks postdeletion, when the rbc become macrocytic with a mean corpuscular volume of $64.3 \pm 11.0 \mathrm{fl}$ vs. $44.1 \pm 3.7 \mathrm{fl}$ and hemoglobin of $6.1 \pm 2.7$ $\mathrm{g} / \mathrm{dl}$ vs. $14.3 \pm 0.6 \mathrm{~g} / \mathrm{dl}$ (Figure 1 ). Up to $60 \%$ of Flvcr1-deleted mice need to be euthanized by 16 weeks after deletion due to severe anemia, with rbc counts below $2.0 \mathrm{M} / \mu \mathrm{l}$ (Supplemental Figure 1; sup- 

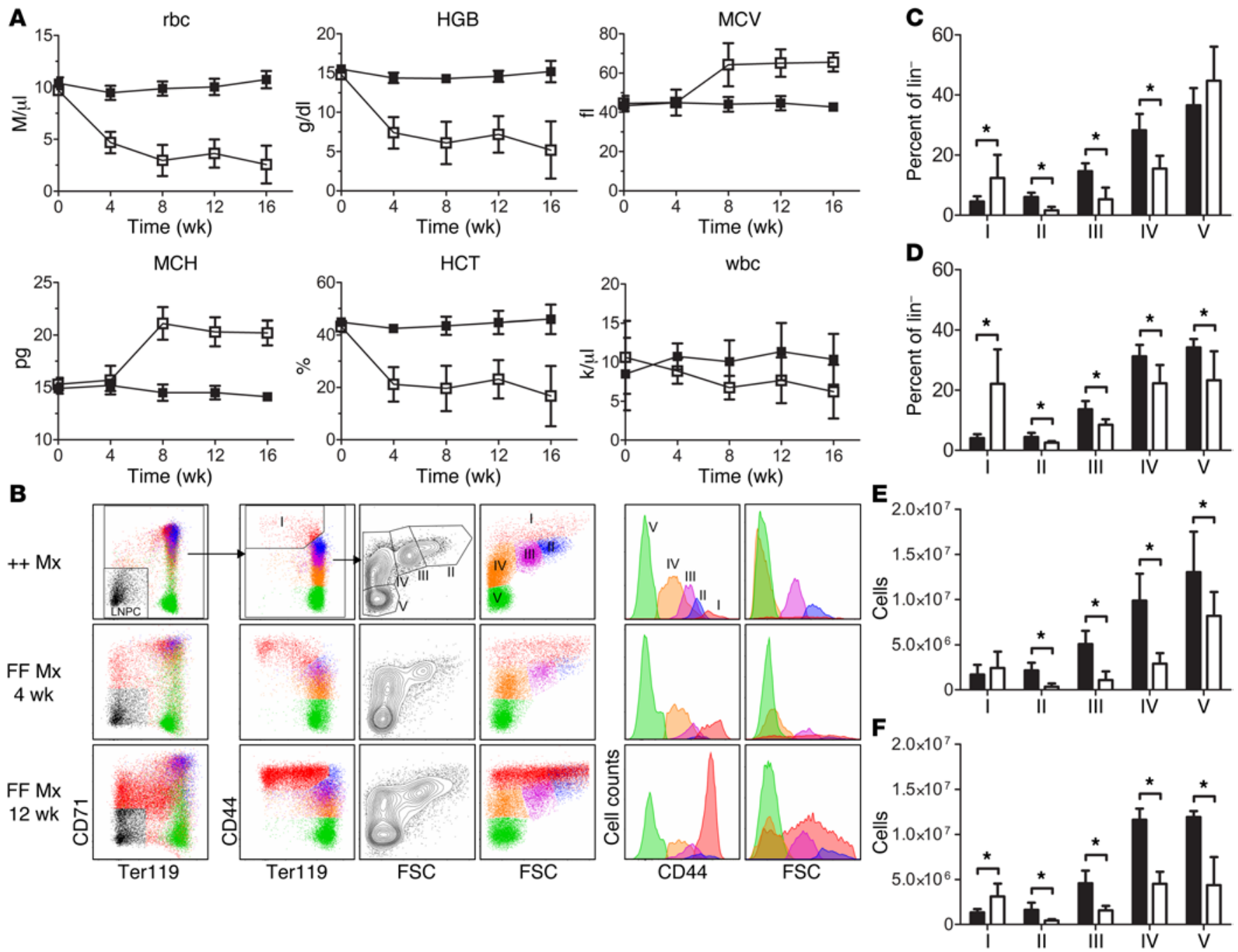

Figure 1. The loss of FLVCR1 results in ineffective erythropoiesis with a CFU-E/proerythroblast block. (A) Peripheral rbc, hemoglobin concentration (HCB), mean corpuscular volume (MCV), mean corpuscular hemoglobin (MCH), hematocrit (HCT), and wbc counts from control (solid squares, $n=6)$ and Flvcr1-deleted (open squares, $n=9$ ) mice were measured before and every 4 weeks after induced deletion for up to 16 weeks. The rbc, HCB, MCV, MCH, and HCT values in Flvcr-deleted mice significantly differ from the values in control mice. Student's $t$ test: $P<0.003$. (B) Representative flow plots from control (++ Mx) and Flvcr1-deleted (FF Mx) cells from femurs and tibias were isolated either 4 or 12 weeks after deletion, as indicated. Lin- (B220-Cr1-CD11b-) marrow cells were analyzed for CD71 and Ter119 to identify committed erythroid cells and LNPC, which include stem cells and progenitors of all hematopoietic lineages. Marrow erythroid cells were gated, as shown in the first 3 columns, to identify each developing erythroid cell population (I, CFU-E/proerythroblasts; II, basophilic erythroblasts; III, polychromatic erythroblasts; IV, orthochromatic erythroblasts and reticulocytes; V, reticulocytes and rbc). The right 3 columns are overlays of all gated erythroid cells presented as CD44 by size (forward scatter, FSC), dot plots, and histograms of CD44 and FSC to show their size and distribution. (C-F)The frequency of developing erythroblasts as a percent of lin cells 4 (C) or 12 weeks (D) after deletion, and the absolute numbers of erythroblasts in femur and tibias 4 (E) and 12 weeks (F) after deletion from control (solid bars) and Flvcr1-deleted (open bars) mice. The 4-week data is from 6 mice each, and the 12 -week data is from 4 mice each. Student's $t$ test: ${ }^{*} P \leq 0.05$. All $P$ values are listed in Supplemental Tables 2 and 3.

plemental material available online with this article; doi:10.1172/ JCI83054DS1), so we restricted most subsequent studies of mice up to 12 weeks after deletion to avoid selection bias with less symptomatic mice. Consistent with the anemia observed, Flvcr1-deleted mice contained fewer than $50 \%$ of the absolute numbers of erythroid precursor cells in femurs and tibias compared with control mice by 12 weeks after deletion (control: $3.15 \pm 0.37 \times 10^{7}$; deleted: $1.46 \pm 0.38 \times 10^{7} ; n=4,4$, respectively; $P<0.001$ ).

Existing flow cytometry methods fail to delineate the early erythroid progenitor population expanded in Flvcr1-deleted mice. Utilizing Ter119, CD44, and cell size instead of CD71 and Ter119 allows identification of discrete erythroid progenitor stages from proerythroblast to mature $\mathrm{rbc}(18-21)$. The earliest erythroid progenitors - burst-forming unit, erythroid (BFU-E) and colony-forming unit, erythroid (CFU-E) - reside in the lineage negative (lin-) $\mathrm{CD}^{-} 1^{+}$Ter119- (glycophorin $^{-}$) populations of mouse BM, fetal liver, and cultured human marrow (22-24). We have previously shown that there is a relative expansion of $\mathrm{BM} \mathrm{lin}^{-}$ CD71+Ter119 ${ }^{-}$and Ter119 ${ }^{10}$ cells in Flvcr1-deleted mice (13). Since the staining panel developed by Chen et al. $(20,21)$ excludes Ter119- cells, we modified the analysis to include these earlier progenitors (lin-CD71+ ${ }^{+}$Ter119-) in population I. We then compared results using the approach of Chen et al. to results using our modified protocol, which includes $\mathrm{CD} 71^{+}$erythroid precursors in ery- 

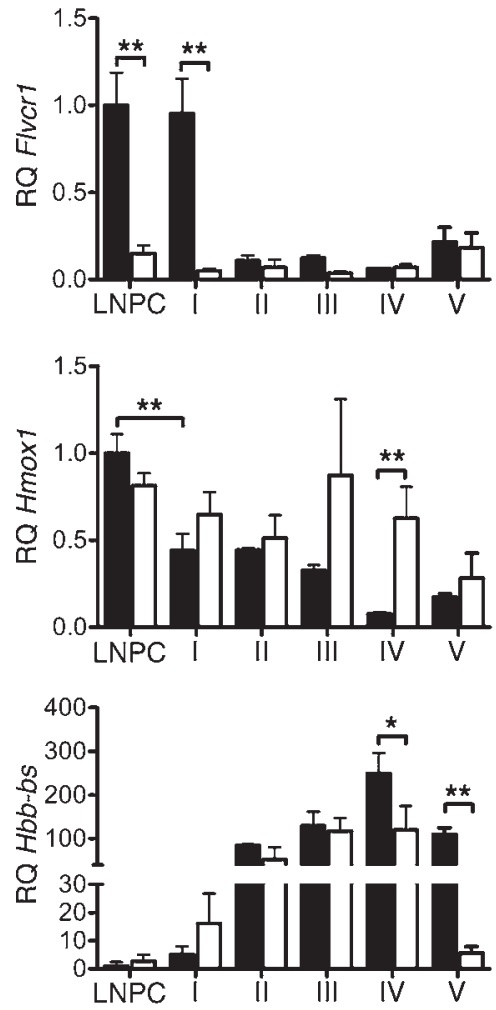
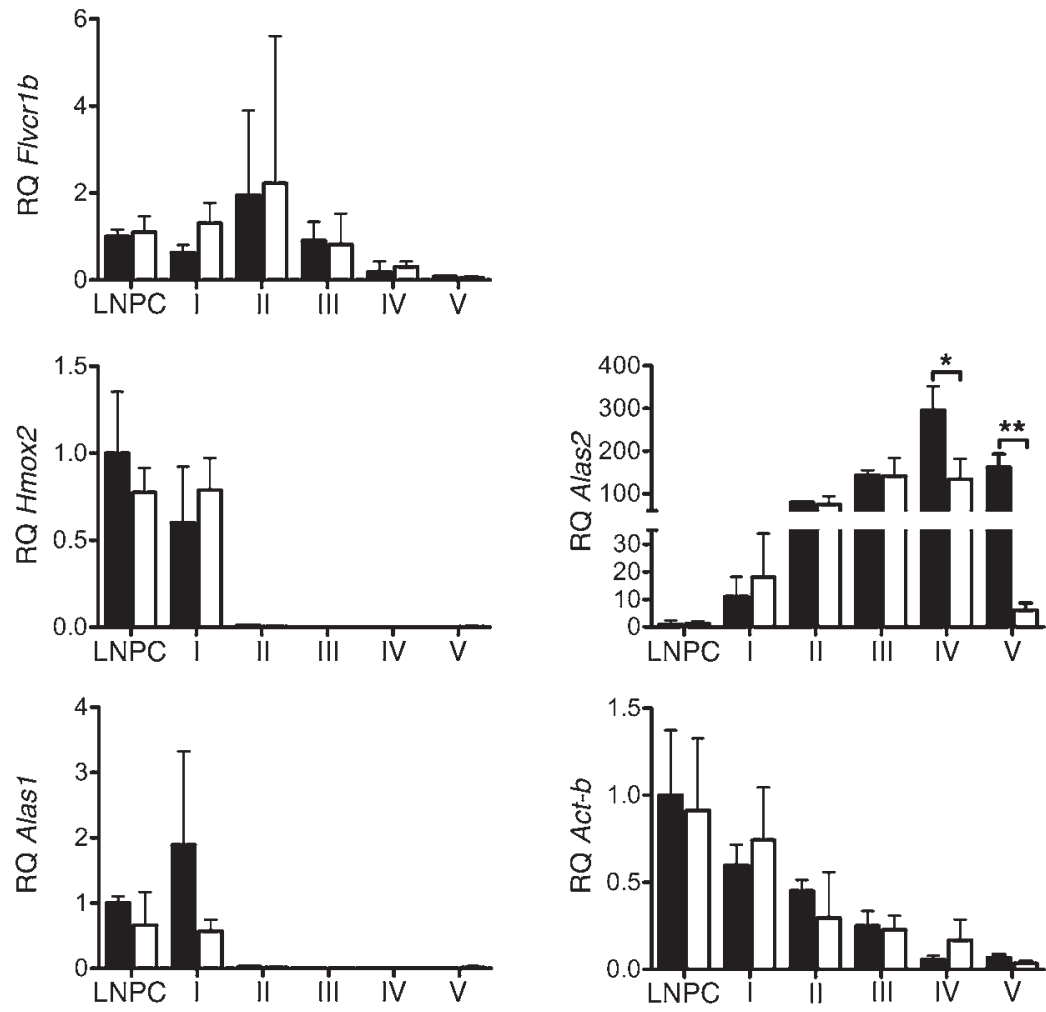

Figure 2. CFU-E/proerythroblasts upregulate heme biosynthesis more than globin synthesis. Relative expression of Flvcr1, Flvcr1b, Hmox1 and 2, Alas1 and $2, \beta$-globin $(\mathrm{H} b b-b \mathrm{~s})$, and $\beta$-actin (Act-b) in sorted marrow erythroblast populations (I-V) quantified by real-time PCR. Mean \pm SD of 3 independently sorted samples are presented from control (solid bars) or Flvcr7-deleted (open bars) mice relative to expression levels in the LNPC from control mice. Of note, the Flvcr1 probe detects total Flvcr1, which includes functional Flvcr1a and Flvcr1b transcripts containing exon 3, while the Flvcr1b probe detects all Flvcr1b transcripts, regardless of whether they encode functional proteins. Normalization of expression levels is described in the Methods. Each sorted sample was derived from a pool of marrow from 3 mice; thus, each bar includes data from 9 mice. Student's $t$ test: ${ }^{*} P<0.05,{ }^{* *} P<0.01$.

throblast population I, regardless of the level of Ter119 expression (Figure 1B and Supplemental Figure 2). The lin ${ }^{-} \mathrm{CD} 71^{+}$Ter119- ${ }^{-}$cells are uniformly CD44 high and have the same broad size distribution as the $\mathrm{Ter} 19^{+}$proerythroblast population. The $\operatorname{lin}^{-} \mathrm{CD} 71^{+} \mathrm{Ter} 119$ to low cells (population I) contain some BFU-E, all CFU-E, and many proerythroblasts (Supplemental Table 1 and Supplemental Figure 3). In healthy control mice, the modified protocol increased the number of erythroid precursors identified in population I by $23.8 \% \pm 10.8 \%$, resulting in the identification of $1.2 \% \pm 0.6 \%$ more erythroid cells in all combined erythroblast populations. However, in Flvcr1-deleted mice, the modified protocol increased the number of erythroid precursors identified in population I by $42.8 \% \pm$ $12.7 \%$, resulting in the identification of $11.3 \% \pm 1.6 \%$ more erythroid cells. Thus, including lin $^{-} \mathrm{CD}^{-} 1^{+} \mathrm{Ter} 119^{-}$cells is essential when evaluating ineffective erythropoiesis, as these early erythroid progenitors may be greatly expanded.

FLVCR1 is required for CFU-E/proerythroblast to basophilic erythroblast differentiation. To further characterize the role of FLVCR1 in erythroblast survival, we evaluated erythroid differentiation in control and Flvcrl-deleted mice under acute conditions (4 weeks after deletion) and under chronic, steady-state conditions (12 weeks or longer after deletion). At both times, there is an increase in the frequency and absolute numbers of CFU-E/proerythroblasts (population I), along with a subsequent reduction in the frequency and absolute numbers of erythroblasts in later stages of development in the Flvcr1-deleted mice, demonstrating a developmental block at the CFU-E/proerythroblast to basophilic erythroblast stage (Figure 1 and Supplemental Tables 2 and 3). This block is much more evident at or after 12 weeks postdeletion, as there is nearly a 3-fold increase in population I erythroblasts compared with control mice. Serum erythropoietin (EPO) levels increase over time with the severity of the anemia (control: $384 \pm 181 \mathrm{pg} / \mathrm{ml}$; 4 weeks: 10,552 $\pm 1,896$ pg/ml; 12 weeks: $51,269 \pm 22,560 \mathrm{pg} / \mathrm{ml} ; n=6,3,3$, respectively; all pairwise $P$ values $<0.03)$. Thus, EPO is the most likely cause of increased numbers of CFU-E/proerythroblasts. Even with this significant increase in $\operatorname{lin}^{-} \mathrm{CD} 71^{+} \mathrm{Ter} 119^{-/ / \mathrm{lo}}$ precursors entering the erythroid lineage under EPO drive, significantly fewer erythroblasts survive through the basophilic erythroblast stage (Figure 1). This developmental block in Flvcr1-deleted mice implies that FLVCR1 is necessary for erythroid differentiation beyond the CFU-E/proerythroblast stage. Marrow culture studies confirm these findings, as the CFU-E/proerythroblasts from Flvcr1-deleted mice generate too few hemoglobinizing cells to be scored morphologically as erythroid colonies; thus, CFU-E are not detectable (Supplemental Table 1 and ref. 13). Identical marrow culture findings are observed in cats viremic with feline leukemia virus subgroup C (FeLV-C) where FLVCR is targeted for destruction $(25,26)$.

Heme synthesis is upregulated prior to substantial globin production. The requirement for FLVCR1 at the CFU-E/proerythroblast stage suggests these cells are uniquely sensitive to heme. To address 

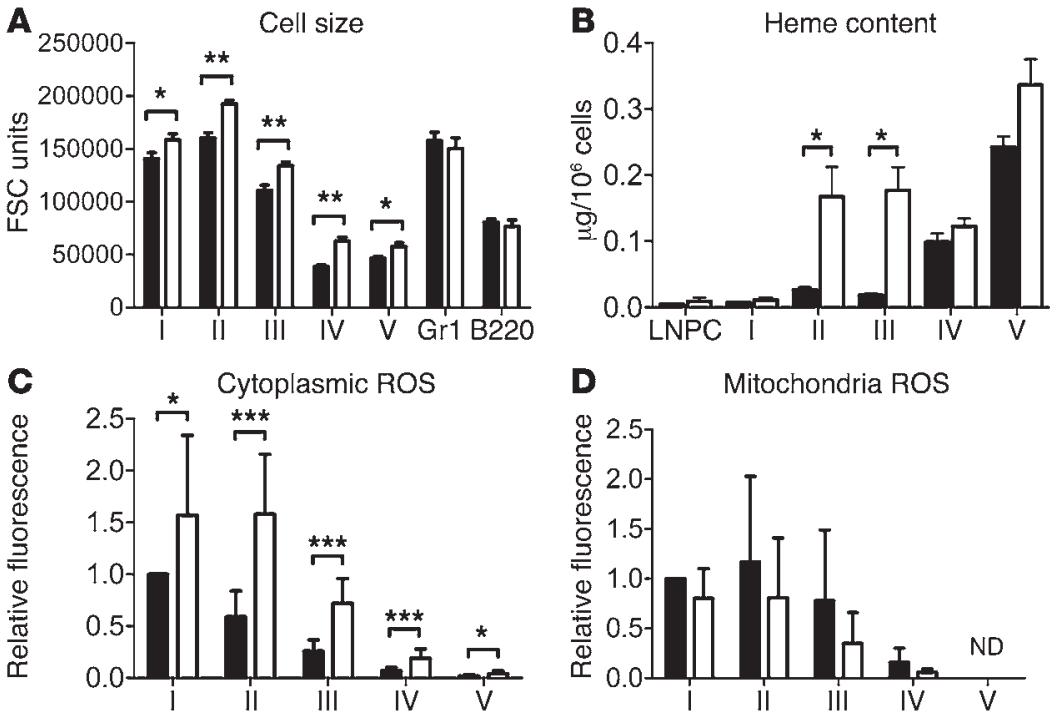

Figure 3. FLVCR1 is necessary throughout erythroid development to maintain heme balance and reduce ROS levels. (A) The relative size of developing erythroblasts (I-V), granulocytes (Gr1), and B cells (B220) in control (solid bars, $n=3$ ) and Flvcr1-deleted (open bars, $n=3$ ) mice 12 weeks after deletion. (B) Heme content of sorted LNPC and committed erythroid progenitors (populations I-V) from control (solid bars) and Flvcr1-deleted (open bars) mice. Mean \pm SD of 3 independently sorted samples. Each sample is from a pool of marrow cells from 3 mice. (C) Relative CM-H2DCFCA fluorescence indicating cytoplasmic ROS in each erythroblast population 4-12 weeks after Flvcr1-deletion from control (solid bars, $n=10$ ) and Flvcr1-deleted (open bars, $n=10$ ) mice. Fluorescence data from multiple independent flow studies were normalized by dividing the fluorescence of each sample population by the mean fluorescence of population I from the control mice. Normalized data from multiple flow studies were then combined for this analysis. (D) Relative MitoSOX Red fluorescence indicating mitochondria ROS in each erythroblast population 4-12 weeks after Flvcr1-deletion from control (solid bars, $n=6$ ) and Flvcr1-deleted (open bars, $n=6$ ) mice. ND, insufficient mitochondrial staining present. Fluorescence data from multiple independent flow studies were normalized as in C. Student's $t$ test: ${ }^{*} P<0.05,{ }^{* *} P<0.01,{ }^{* *} P<0.001$.

this, we evaluated gene expression of ALAS, globin, HMOX, and FLVCR1 throughout erythroid differentiation. Peak expression of Alas 2 and $\beta$-globin (Hbb-bs) occurs at the orthochromatic and reticulocyte stage (population IV) of erythroid differentiation when Hmox1, Hmox2, and Flvcr1 expression are at their lowest (Figure 2). However, we are interested in what is occurring around the CFU-E/proerythroblast stage. As cells differentiate from a lin precursor cell (LNPC) to CFU-E/proerythroblast stage (population I), Alas 2 is significantly upregulated ( $>10$-fold), while Alas 1 is still highly expressed and $\beta$-globin transcription initiates (Figure 2). Thus, the CFU-E/proerythroblast stage is when erythroid heme synthesis initiates. Since heme is required for both globin transcription and translation $(7,8), \mathrm{CFU}-\mathrm{E} /$ proerythroblasts would be subject to heme-mediated damage until adequate globin protein is produced, unless excess heme is degraded or eliminated. During this same transition, Hmox1 and Hmox2 are both downregulated about $50 \%$, significantly reducing that capacity of heme degradation, while Flvcr1 expression is not significantly reduced (Figure 2). Therefore, CFU-E/proerythroblasts preferentially utilize FLVCRmediated heme export to eliminate excess heme. Since FLVCR1 has a relatively long half-life (27) - and during human erythroid differentiation, the FLVCR1 mRNA levels peak before protein levels (12) - the measured Flvcr1 mRNA levels may underestimate the actual levels of FLVCR1 protein in subsequent developmental stages. The lack of a specific antibody for FLVCR1 in mouse samples prevents direct analysis of FLVCR1 protein. During the CFU-E/proerythroblast (population I) to basophilic erythroblast (population II) transition, Alas 2 is further upregulated, along with significant upregulation of $\beta$-globin mRNA and complete loss of Hmox 2 expression (Figure 2). Thus, heme synthesis is substantially upregulated during the CFU-E/proerythroblast stage, when HMOXs are low or absent and Flvcr1 is still highly expressed. Expression of Flvcr1b-specific transcripts encoding the mitochondrial isoform peak in population II and are mostly gone in population IV, when Alas2 and $\beta$-globin are expressed the highest. This suggests that other mitochondrial heme exporters may play an important role in erythroid differentiation during peak heme production.

In Flvcr1-deleted mice, there are alterations in expression of both heme synthesis and degradation genes during erythroid differentiation compared with control mice. While the expression of functional Flvcr1 isoforms (those containing exon 3) are ablated in the cell populations that normally express them as expected, there are no apparent alterations in Flvcr $1 b$-specific transcript levels in any population using a probe directed at its unique $5^{\prime}$ untranslated region (14). However, these Flvcrlb transcripts will lack exon 3 and result in nonfunctional proteins. Hmox1 is slightly elevated in CFU-E/proerythroblasts (population I) and significantly increased in the late stages, where it is normally not expressed. The most likely reason for this is elevated heme levels. However, it could be in response to other oxidative stresses. There is also a substantial reduction in Alas1 expression in population I erythroblasts of Flvcr1-deleted mice, which would be consistent with heme-mediated inhibition of Alas1 transcription in these cells (3). Both Alas 2 and $\beta$-globin expression are significantly reduced in populations IV and V, when they are normally expressed at high levels (Figure 2). Since Alas 2 transcription is regulated by iron but not heme $(4,5)$, the reduction of Alas 2 transcription most likely reflects selection for cells that synthesize less heme and are, thus, capable of surviving in the absence of FLVCR1. The reduction in Alas 2 and reduced heme synthesis would be expected to result in less $\beta$-globin transcription, since $\beta$-globin transcription is at least in part regulated by heme levels (7). Thus, these data suggest that the loss of FLVCR1 results in increased heme levels, increased heme degradation in CFU-E/ proerythroblasts, and subsequently, preferential survival of cells that make less heme to later stages of differentiation.

FLVCR1-deficient erythroblasts die from excess heme. Since mature rbc in Flvcr1-deleted mice are macrocytic with elevated mean corpuscular heme, we wanted to determine if these alterations are concomitant with the developmental block observed in erythroblasts. There is a significant increase in erythroblast size in all Flvcr1-deleted erythroblasts, including marrow reticulocytes and mature erythroid cells (Figure 3A), consistent with the macrocytosis observed in peripheral rbc. The increased cell size 

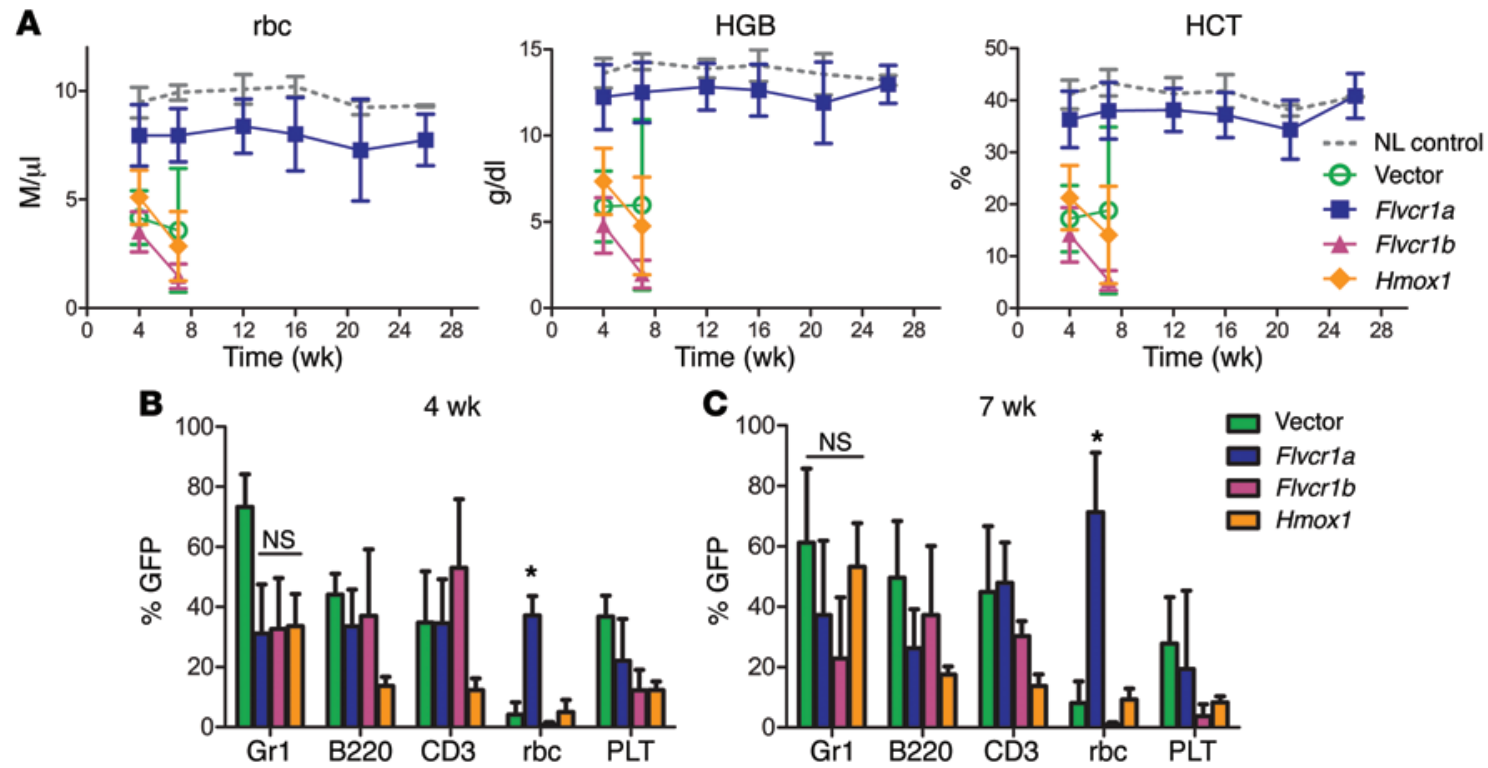

Figure 4. Excess cytoplasmic heme, but not mitochondrial heme, causes erythroid failure in Flvcr1-deleted mice. (A) Peripheral rbc counts, hemoglobin (HCB), and hematocrit (HCT) levels in transplanted mice that received normal marrow (NL Control, $n=9$ ) or Flvcr1-deleted marrow that had been transduced with empty vector $(n=9)$ or vectors expressing Flvcr1a $(n=15)$, Flvcr1b $(n=8)$, or Hmoxi $(n=10)$. (B and C) The frequency of the rbc, platelets (PLT), and the donor-derived peripheral granulocytes (Gr1), B cells (B220), and T cells (CD3), which are positive for the GFP transduction marker at 4 weeks (B) and 7 weeks (C) after transplant with Flvcr1-deleted marrow transduced with empty vector, Flvcr1a, Flvcr1b, or Hmox1 as indicated. Student's $t$ test: ${ }^{*} P<0.001$.

is limited to the erythroid lineage, as neither granulocytes nor B lineage cells from Flvcr1-deleted mice are larger, and the morphology of sorted erythroblasts is not altered, other than being slightly larger (Supplemental Figure 3). While the presence of macrocytic erythroblasts is consistent with elevated heme levels as observed in mature rbc, it does not directly demonstrate that Flvcr1-deleted erythroblasts have elevated heme.

To directly determine whether the erythroid failure in Flvcr1deleted mice is due to a loss of heme export via FLVCR1 and, consequently, an increase in intracellular heme, we sorted developing erythroblasts and measured total cellular heme content from pooled marrow samples. The heme content in Flvcr1deleted erythroblasts is significantly elevated compared with control erythroblasts in populations II and III, but not in LNPC, which includes progenitor and stem cells of all hematopoietic lineages (Figure 3B). In normal control erythroblasts, heme content increases at the orthochromatic stage (population IV) when Alas 2 and $\beta$-globin are most highly expressed and Flvcr1 and Hmox1 are both expressed the least (see Figure 2). We completed additional studies using marrow samples from individual mice by combining populations I and II into a single collection gate, and we obtained essentially identical results with a larger sample number (Supplemental Figure 4), consistent with the CFU-E/ proerythroblast to basophilic erythroblast block observed in these mice. Since FLVCR1 exports planar porphyrins (late heme precursors) in addition to heme (28), we evaluated protoporphyrin IX (PPIX) content in Flvcr1-deleted erythroblasts and found that PPIX content was slightly elevated in all erythroblast populations (Supplemental Figure 5). Thus, FLVCR-deficient erythroblasts accumulate PPIX, in addition to heme. This result may reflect that PPIX is routinely exported by FLVCR (28), or alternatively, excess heme in Flvcr1-deleted erythroblasts exceeds apo-hemoprotein capacity, thus, interfering with ferrochelatase activity (29) and resulting in increased PPIX levels.

Since heme is a powerful driver of oxidative damage, we next evaluated ROS levels in developing erythroblasts from control and Flvcr1-deleted mice using the ROS sensor CM-H2DCFCA. In Flvcr1-deleted marrow, there are significant increases of ROS in all erythroblast populations (Figure 3C), concordant with the elevated heme levels observed. Even CFU-E/proerythroblasts had a significant elevation in ROS, even though heme was only marginally elevated. As CFU-E/proerythroblasts express very low levels of globin (Figure 2), they would be most sensitive to elevated heme levels, which is consistent with these observations. As an alternative ROS sensor, we utilized CellROX Deep Red, which did not detect any alteration in ROS levels in Flvcr1-deleted erythroblasts relative to control erythroblasts. Furthermore, it did not indicate increased ROS in response to $10 \mu \mathrm{M} \mathrm{H}_{2} \mathrm{O}_{2}$, while CM-H2DCFCA provided a 7-fold increase in fluorescence intensity in marrow cells. This would suggest that the cellular ROS signal we detected is dependent upon $\mathrm{H}_{2} \mathrm{O}_{2}$ or other peroxides. This is consistent with published results indicating heme sensitizes cells to $\mathrm{H}_{2} \mathrm{O}_{2}$ and peroxide damage (10).

Since the FLVCR1b isoform is a mitochondrial heme exporter, we evaluated mitochondrial ROS in Flvcr1-deleted erythroblasts to determine the role of mitochondrial heme in the erythroid failure of Flvcr1-deleted mice using MitoSOX Red. There was no elevation in mitochondrial ROS detected at any stage, and all erythroblasts from Flvcr1-deleted mice have reduced mitochondrial ROS staining with MitoSOX Red (Figure 3D), suggesting there may be a loss of mitochondrial integrity. The loss of mitochondrial integrity may be a primary defect or secondary to elevated cytoplasmic heme and cytoplasmic ROS. Treatment of marrow cells with $10 \mu \mathrm{M} \mathrm{H}_{2} \mathrm{O}_{2}$ resulted in 
A

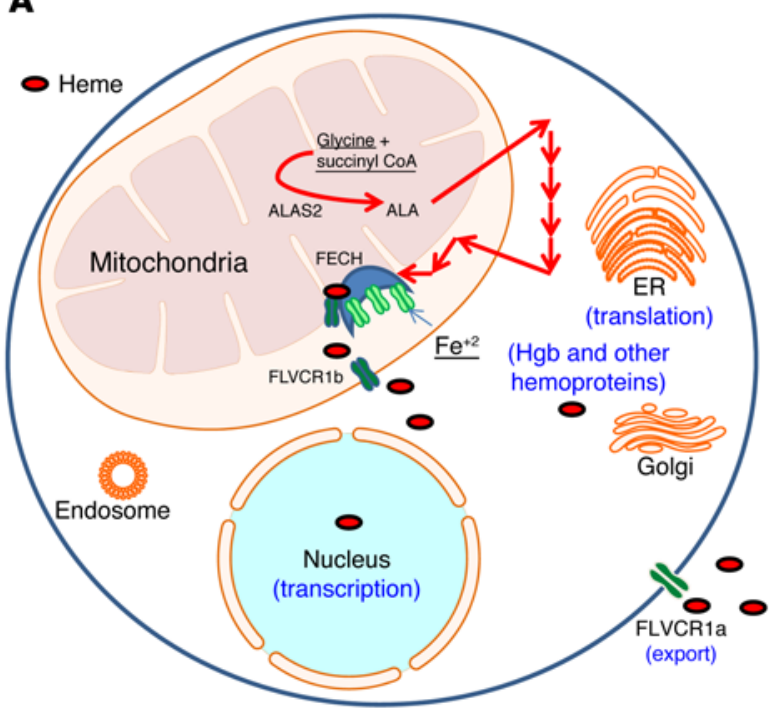

C

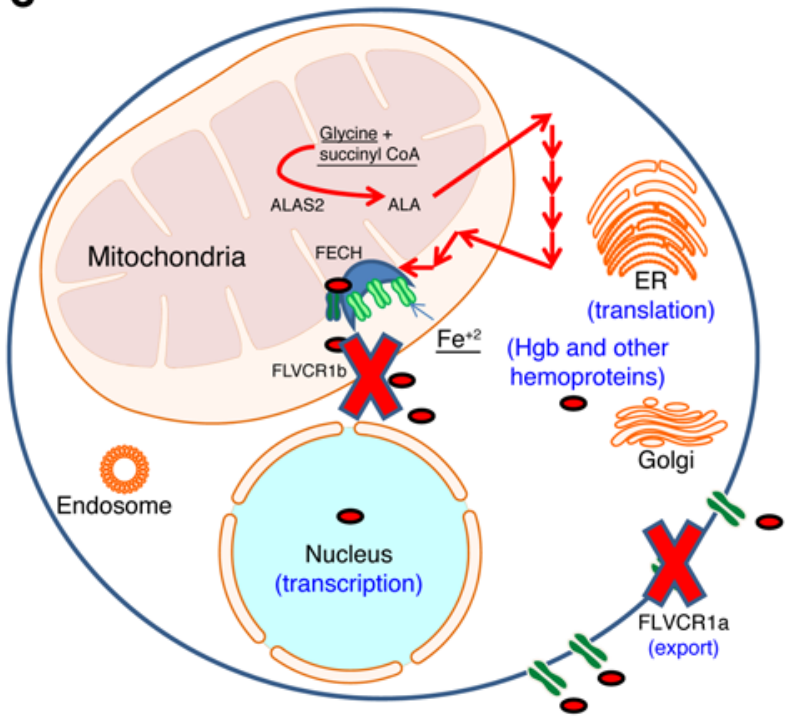

B

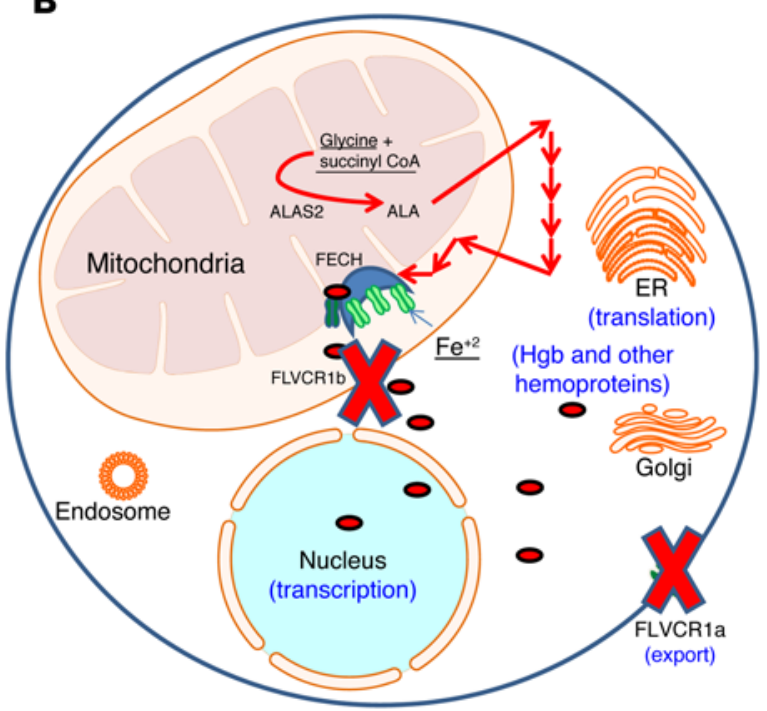

Figure 5. The role of FLVCR1 in the coordination of heme and globin during erythroid differentiation. (A) When erythroid cells reach the CFU-E/proerythroblast stage, transferrin receptor 1 (Tfrc, CD71) is upregulated, transferrin-iron enters the cells, and heme synthesis initiates as ALAS2, the erythroid-specific initial step of the heme synthetic pathway, has a $5^{\prime}$ iron-responsive element (IRE) preventing translation in the absence of sufficient iron. Heme then induces globin production, since it is a transcriptional and translational regulator of globin synthesis. Thus, there is an obligate time when heme is present but globin protein is insufficient. During this interval, FLVCR1a is required to export surplus heme. (B) Deletion of both isoforms of FLVCR1 results in the accumulation of intracellular heme in excess of cellular needs, resulting in cytoplasmic ROS and erythroblast death, which leads to anemia. (C) Subsequent expression of FLVCR1a on the cell surface restores normal cytoplasmic heme export and erythroblast differentiation, while expression of the mitochondrial isoform FLVCR1b does not (see Figure 4). Thus, the anemia in Flvcr1-deleted mice results from proerythroblast death caused by excessive cytoplasmic heme and ROS. an average $10 \%$ reduction in MitoSOX Red fluorescence, similar to what we observed in Flvcr1-deleted erythroblasts. This is consistent with elevated cytoplasmic heme and cytoplasmic ROS, resulting in reduced mitochondrial integrity. Since heme sensitizes cells to $\mathrm{H}_{2} \mathrm{O}_{2}$ and peroxide damage (10), the elevated heme levels in Flvcr1-deleted erythroblasts may lead to peroxide damage and cell death. We previously demonstrated that Flvcr1deleted $\mathrm{CD} 1^{+}$erythroblasts die via apoptosis (30), and with further delineation of erythroblast development in Flvcr1-deleted mice, we found increased cell death at all stages of erythroid differentiation (Supplemental Figure 5).

The erythroid defect in Flvcr1-deleted mice is caused by the absence of cytoplasmic heme export. The Flvcr1-deletion disrupts both FLVCR1a and FLVCR1b transcripts, resulting in nonfunctional proteins $(13,14)$. The low expression of Flvcr $1 b$ during peak heme production and the presence of elevated cytoplasmic but not mitochondrial ROS suggests it is not the loss of mitochondrial heme export, but rather cellular heme export, that drives the erythroid cell death in Flvcr1-deleted mice. To directly test this, we reconstituted mice with Flvcr1-deleted marrow transduced with a retroviral vector encoding either FLVCR1a (expressed on the cell membrane) or FLVCR1b (expressed on the mitochondria) to determine the role each isoform plays during erythropoiesis. Mice transplanted with marrow cells expressing FLVCR1a remained alive and healthy with normal hematocrits and hemoglobin levels at least 6 months after transplantation and had a median survival of more than 35 weeks. Conversely, mice transplanted with marrow cells expressing FLVCR1b die with severe anemia by 10 weeks after transplant and have a median survival time of 7 weeks, which is comparable to mice that receive marrow cells transduced with a control vector encoding only GFP (Figure 4 and Supplemental Figure 6). There were no differences in the marking frequency of white cell populations between mice that received either FLVCR1a- or FLVCR1b-expressing marrow (Figure 4), and there were no differences in the levels of GFP protein, reflecting equivalent mRNA levels (Supplemental Table 4). However, FLVCR1a-expressing cells contribute to the rbc compartment, while the FLVCR1b-expressing cells do not. This 
A
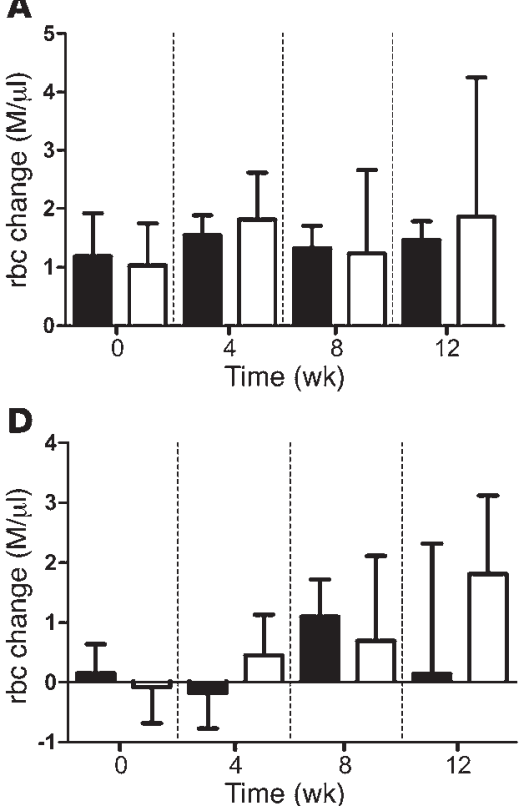

B

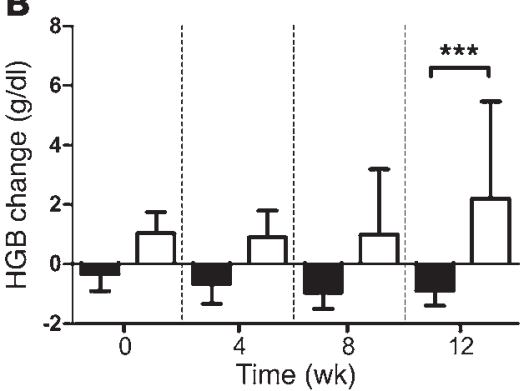

E

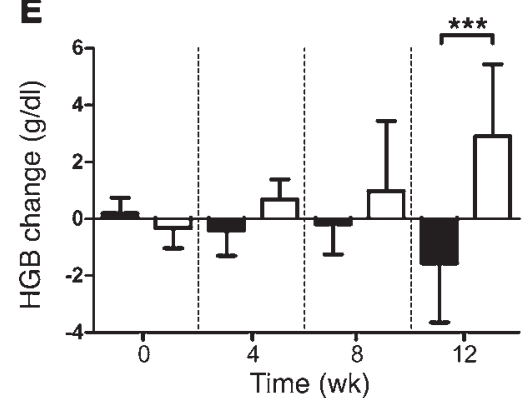

C

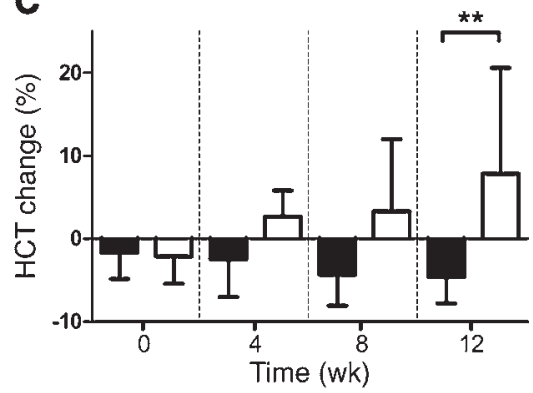

$\mathbf{F}$

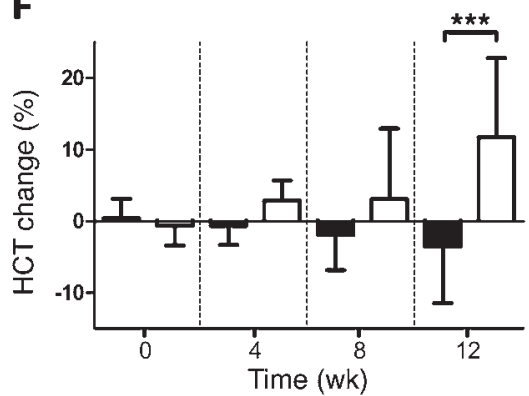

Figure 6. Iron restriction improves the anemia in mice with erythroblast heme overload. (A-C) The difference in peripheral rbc counts (A), hemoglobin levels (HCB) (B), and hematocrits (HCT) (C) in Tfrc ${ }^{+/-} ;$Mxcre mice compared with the mean value of Tfrc ${ }^{+/+} ;$Mxcre mice (solid bars, $\left.n=10\right)$ and Tfrc ${ }^{+/-} ;$Flvcr1deleted mice compared with the mean value of Tfrc ${ }^{+/+}$; Flvcr1-deleted mice (open bars, $\left.n=7\right)$. (D-F) The difference in peripheral rbc counts (D), HCB (E), and HCT (F) in Mxcre mice fed an iron-deficient diet compared with the mean value of Mxcre mice fed a normal diet (solid bars, $n=10$ ) and in Flvcr1deleted mice fed an iron-deficient diet compared with the mean value of Flvcr1-deleted mice fed a normal diet (open bars, $n=11$ ). CBC measurements are presented in Supplemental Figures 9 and 10. One-way ANOVA Tukey-Kramer test: ${ }^{* *} P<0.01,{ }^{* *} P<0.001$.

demonstrates that cytoplasmic heme export by FLVCR1a, but not mitochondrial heme export by FLVCR1b, is able to support erythropoiesis in mice lacking both FLVCR1 isoforms (Figure 5).

To directly test whether heme degradation via HMOX could overcome the need for FLVCR1a during erythroid differentiation, we overexpressed Hmox1 in Flvcrl-deleted marrow and then transplanted these cells into irradiated recipient mice. We found that Hmox 1 expression did not overcome the need for functional FLVCR1a in erythroblasts despite having marking frequency in granulocytes comparable with that of FLVCR1a-transduced marrow (Figure 4 and Supplemental Figure 6) and comparable levels of mRNA, as reflected by equivalent GFP levels (Supplemental Table 4). Furthermore, we demonstrated the vector produces high levels of HMOX1 protein in mouse cells (Supplemental Figure 7). The lack of marking among rbc indicates HMOX1 is not able to replace the loss of FLVCR1 in developing erythroblasts. To determine if overexpressed HMOX1 was capable of functioning in erythroblasts, we sorted lin $^{-} \mathrm{CD}_{71^{+}}$erythroblasts (populations I-IV) from these mice into $\mathrm{HMOX1}^{-}\left(\mathrm{GFP}^{-}\right)$and $\mathrm{HMOX1}^{+}\left(\mathrm{GFP}^{+}\right)$populations. The level of heme in these cells was $0.31 \pm 0.1 \mu \mathrm{g}$ per $10^{6}$ cells and $0.14 \pm 0.1 \mu \mathrm{g}$ per $10^{6}$ cells, respectively: an approximate 2-fold reduction in cellular heme in cells expressing HMOX1. While we were unable to further delineate which stage of erythroblasts had functional HMOX in these transplant studies because of the paucity of erythroblasts, it is clear that overexpressed HMOX1 can function in erythroblasts; however, HMOX1 is not capable of degrading heme sufficiently to overcome the need for FLVCR1a in early erythroblasts. It is unclear whether HMOX1 is not functional in early erythroblasts or if newly synthesized heme is not being delivered to HMOX1 for degradation in these cells.
The reduction of heme synthesis improves erythropoiesis in Flvcr1deleted mice. Since direct heme degradation was ineffective, we took an alternative approach and tested whether interference with the heme biosynthetic pathway could ameliorate the anemia in FLVCR1-deficient mice. We first bred Flverflff; Mxcre mice to ferrochelatase mutant mice to make them functionally heme deficient. However, ferrocheletase (Fech) mutant mice have increased levels of reactive porphyrins, which build up in Flvcr1deleted mice $(28,31)$. Consequently, rbc parameters in double mutant mice did not significantly differ from rbc parameters in Flvcr1-deleted mice (Supplemental Figure 8).

As a preferable strategy to reduce iron availability for heme synthesis, we bred Flvcrl/fl; Mxcre mice to transferrin receptor heterozygous $\left(\mathrm{TfrC}^{+/-}\right)$mice. Similar to Fech-deficient mice, $\mathrm{Tfrc}^{+/-}$ mice have a mild microcytic, hypochromic anemia consistent with iron/heme deficiency $(32,33)$. As iron availability is a key factor in initiating erythroid heme synthesis (29), we tested whether the mild iron restriction in $\mathrm{TfrC}^{+/-}$mice was sufficient to improve erythropoiesis in Flvcr1-deleted mice. While Tfrc ${ }^{+/}$; Mxcre mice start with a higher rbc count and lower hemoglobin and hematocrit than Mxcre control mice, the Tfrc ${ }^{+/}$; Flvcr1-deleted mice have a higher rbc count and also higher hemoglobin and hematocrits than the Flvcr1-deleted mice by 12 weeks after deletion (Figure 6 and Supplemental Figure 9). Thus, the iron restriction mediated by reduced transferrin receptor improves the anemia caused by Flvcr1 deletion during erythropoiesis. We further restricted iron by feeding mice an iron-deficient diet (<6 ppm iron vs. 220 ppm iron) after deleting Flvcrl. This iron-deficient diet reduced the hemoglobin and hematocrit in Mxcre control mice while increasing the hemoglobin and hematocrit in Flvcrl-deleted mice (Figure 6 and 

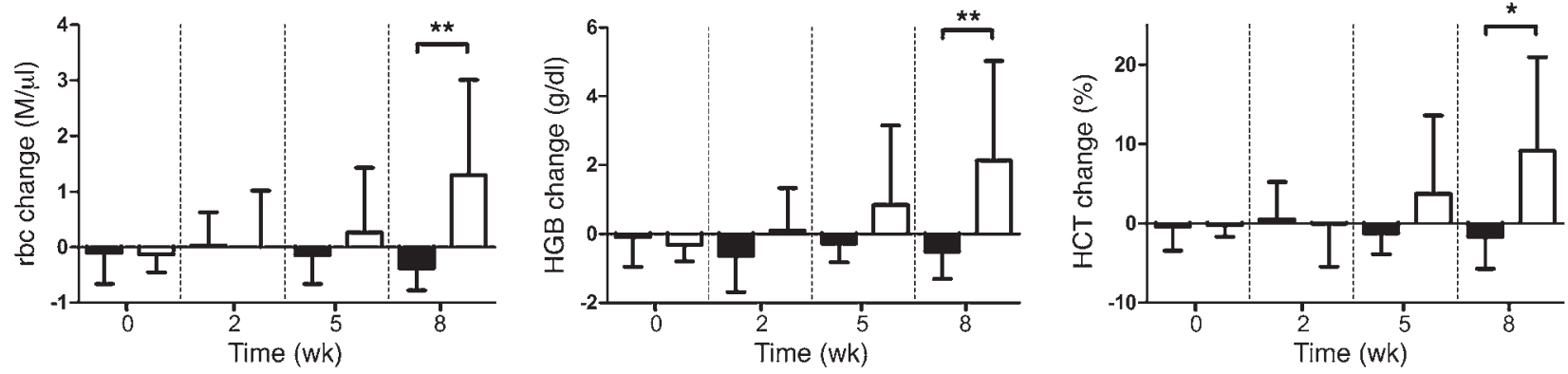

Figure 7. Reducing heme biosynthesis improves the anemia in mice with erythroblast heme overload. The difference in peripheral rbc counts, hemoglobin levels (HCB), and hematocrits (HCT) in Mxcre mice treated with INH for 8 weeks compared with the mean value of Mxcre mice treated with water (solid bars, $n=10$ ) and Flvcr1-deleted mice treated with INH for 8 weeks compared with the mean value of Flvcr1-deleted mice treated with water (open bars, $n=8)$. CBC measurements are presented in Supplemental Figure 11. One-way ANOVA Tukey-Kramer test: ${ }^{*} P<0.05,{ }^{* *} P<0.01$.

Supplemental Figure 10). Thus, restricting iron delivery to the erythron via reduced transferrin receptor expression or an irondeficient diet improves rbc development in mice with elevated erythroblast heme mediated by the loss of FLVCR1.

In addition to altering iron availability for heme synthesis, we treated mice with isonicotinylhydrazine (INH) to inhibit the first step in the heme synthesis pathway: the conversion of succinyl CoA and glycine to 5-aminolevulinic acid by ALAS (34). After 8 weeks of INH treatment, Mxcre mice had a slight reduction in their rbc numbers, hematocrits, and hemoglobin levels compared with control-treated Mxcre mice, while the INH-treated Flvcr1-deleted mice showed increased rbc numbers, hematocrits, and hemoglobin levels compared with control-treated Flvcr1-deleted mice (Figure 7 and Supplemental Figure 11). This improvement in rbc parameters was also accompanied by a significant improvement in survival (Supplemental Figure 11).

\section{Discussion}

Erythroblasts from Flvcr1-deleted mice die at the CFU-E/proerythroblast-to-basophilic erythroblast transition, resulting in severe anemia. The cell death is associated with elevated intracellular heme and elevated cytoplasmic ROS in developing erythroblasts at all stages. Both restricting iron availability and impairing a heme biosynthetic enzyme as 2 experimental approaches to inhibit heme synthesis improve the anemia in Flvcr1-deleted mice, proving that heme excess causes the erythroid marrow failure and that FLVCR1-mediated heme export is required for successful erythroid differentiation prior to heme-dependent upregulation of globin levels.

The major developmental block between the CFU-E/proerythroblast and basophilic erythroblast stages in Flvcr1-deleted mice (Figure 1) suggests these early erythroblasts are particularly sensitive to excess heme and ROS, which correlates to the stages when globin production is absent or just beginning. Additionally, the elevated cytoplasmic ROS and apoptosis throughout all subsequent erythroblast stages in Flvcrl-deleted mice is accompanied with high levels of heme, suggesting ongoing ROS damage, leading to cell death throughout erythroid differentiation. Thus, the imbalance of heme synthesis to heme utilization, degradation, or export at any erythroblast stage may lead to cell death. Recent studies have identified a novel form of iron-dependent cell death in tumors termed ferroptosis (35-37), which may, along with apoptosis, contribute to the demise of Flvcr1-deleted erythroblasts. Ferroptosis is an iron-dependent cell death resulting from elevation of cytosolic and lipid (but not mitochondrial) ROS, which does not include traditional markers of apoptosis such as mitochondrial cytochrome $\mathrm{c}$ release or caspase activation (35). While ferroptosis is not well characterized, it is a relatively slow death occurring over many hours, as opposed to the rapid cell death observed with traditional apoptosis. Since erythropoiesis is a very rapid process in mice, and if the elevated heme-induced ROS causes a slow cell death via ferroptosis, then cell death could occur very early (e.g., CFU-E/proerythroblast stage), accounting for the drop in cell numbers between stages I and II. However, cells continue to die during later stages of erythropoiesis. These kinetics and the presence of sustained cytosolic ROS are supported by our observations.

There have been 2 isoforms of FLVCR1 identified; the long isoform, FLVCR1a, is a broadly expressed cell surface heme exporter, while the short isoform, FLVCR1b, is a mitochondrial heme exporter $(12,14)$. Both the FLVCR1a/b-null and the FLVCR1a-specific null mice fail to survive to birth and display craniofacial and digit abnormalities during development. However, the FLVCR1a-null mice appear to have intact fetal erythropoiesis (13, 14), suggesting that FLVCR1a may not be essential for erythropoiesis. This was tested in a short-term transplant model that failed to observe any defect in mice transplanted with FLVCR1a-deficient fetal liver cells 4 weeks after transplant using CD71 and Ter119 flow analysis (14). Hematocrit and hemoglobin were not reported. There are subtle differences that can be identified by flow cytometry in deleted mice; however, they are not easily observed by CD71 and Ter119 staining 4 weeks after deletion (Figure 1B). Without quantitative $\mathrm{CBC}$ measurements from the FLVCR1a-null transplant, it is not possible to determine if the FLVCR1a-specific null has a unique developmental defect or is completely recapitulated with the combined FLVCR1a/b deletion observed here. The ability of FLVCR1a to completely rescue erythropoiesis in a combined FLVCR1a/b-deleted mouse (Figure 4 and Supplemental Figure 6), however, clearly demonstrates that erythropoiesis in adult mice requires FLVCR1a and not FLVCR1b. Therefore, the cell surface isoform (FLVCR1a), which exports heme from the cell, is essential, while the mitochondrial isoform (FLVCR1b) is not essential (Figure 5) and suggests other mitochondrial heme exporters are functioning during erythropoiesis. This is directly supported by the lack 
of elevated mitochondrial ROS within any erythroid population in Flvcrl-deleted mice (Figure 3D) and low levels of Flvcrlb transcripts during times of peak heme production in normal control mice (Figure 2 and Figure 3B). Since FLVCR1a-deleted mice die in utero with apparently intact fetal erythropoiesis (14), the FLVCR1b isoform could be needed during fetal erythropoiesis.

Overexpression of HMOX1 was unable to compensate for the loss of FLVCR1 (Figure 4 and Supplemental Figure 6), indicating that heme export and heme degradation have distinct roles in handling excess heme during erythropoiesis. Potentially, HMOX1 does not function in early erythroblasts or endogenously synthesized heme does not traffic directly to cytoplasmic HMOX1 in erythroid cells. Our results are consistent with the late onset of anemia observed in HMOX1-deficient mice (38), where the anemia is attributed to poor iron recycling as opposed to ineffective erythropoiesis from heme excess. Alternatively, HMOX1 may degrade heme and release iron, which is then recycled for heme synthesis by rerouting the iron within the cell or by its reuptake (via the transferrin receptor) after ferroportin-mediated export $(39,40)$. Thus, it is possible that heme degradation and subsequent iron export may be a way to maintain the balance between available heme and globin production (11) only during the late stages of erythropoiesis, when the transferrin receptor (CD71) is no longer expressed.

Heme synthesis requires both porphyrin synthesis and iron delivery. We therefore tested ways to interfere with these processes in Flvcr1-deleted mice with the goal of reducing heme synthesis and improving anemia, and we learned that heme and iron regulation is complex. Since transferrin receptor heterozygous mice have reduced iron delivery to the erythron for erythropoiesis (32), we initially hypothesized that Flvcr1-deleted mice that were also haploinsufficient for the transferrin receptor would have reduced heme toxicity and improved erythropoiesis. While these mice still developed severe anemia, suggesting that a modest reduction of heme synthesis is inadequate to overcome the loss of FLVCR1 in early erythroblasts, there was a consistent improvement in the hemoglobin and hematocrit levels (Figure 6, B and C). This contrasted the reduction in hemoglobin and hematocrit seen in control mice (intact Flvcr) that were haploinsufficient for the transferrin receptor. Since EPO regulates transferrin receptor expression $(33,41)$, the increasing levels of EPO observed as the anemia progresses in Flvcr1-deleted mice may counteract the mild iron restriction by increasing transferrin receptor levels in the anemic mice, allowing better scavenging of transferrin-iron. Further restriction of iron delivery to the erythron with an iron-deficient diet also resulted in improved hemoglobin and hematocrits (Figure 6, E and F). Finally, using INH to interfere with ALAS function also improved rbc parameters and led to a significant improvement in viability (Figure 7 and Supplemental Figure 11). Thus, interfering with heme synthesis either via reducing porphyrin synthesis or restricting iron availability for erythropoiesis significantly improves, but does not completely correct, the anemia of mice lacking FLVCR1. This further validates the concept that FLVCR1 is essential for effective erythropoiesis because it reduces early erythroblast heme levels when alternative methods to detoxify heme are either not present or nonfunctional.

Our studies imply that the production of hemoglobin requires the balance between the enzymatic synthesis of heme and the transcription and translation of globin to assure that heme is always available but never in excess. By extension, normal (unbridled) heme production coupled with a slowed initiation of globin transcription or translation could result in a clinical outcome similar to that seen in Flvcr1-deleted mice. The finding that Diamond Blackfan anemia and the macrocytic anemia that characterizes myelodysplasia with an isolated deletion of chromosome $5 q$ (del[5q] MDS) result from haploinsufficiencies of ribosomal proteins and poor ribosome assembly $(42,43)$ may be a relevant human pathophysiology. When ribosome function is impaired, erythroblast globin production would be impaired, resulting in quantities of free heme that exceed the export capacity of FLVCR1, leading to erythroblast apoptosis and anemia similar to Flvcr1-deleted mice.

Thalassemia is also associated with ineffective erythropoiesis, but its pathogenesis is more complex. Although globin synthesis should initiate normally, globin transcription is reduced, which could lead to heme excess. This early erythropoiesis insult, however, would be greatly compounded by the imbalance of $\alpha$ and $\beta$ globin chains and later erythroid cell death. Interventions such as apotransferrin administration, increasing hepcidin, and reducing TMPRSS6 (all of which could restrict iron delivery to the erythron) improve rbc production in $\beta$-thalassemia $\left(\mathrm{Hbb}^{t h 3 /+}\right)$ mice $(44-47)$, which supports the concept that heme excess plays a role and suggests that our observations in Flvcr1-deleted mice may be relevant to this disorder, as well. Finding a way to reduce heme synthesis or improve FLVCR1 function may improve the ineffective erythropoiesis of these disorders.

\section{Methods}

Animals and transplant studies. Flvcrlfff; Mxcre mice with a conditional deletion of both FLVCR1 isoforms were previously described (13). $\mathrm{ffrc}^{+/-}$ (32) were obtained from Nancy Andrews (Duke University, Durham, North Carolina, USA) and Fech mutant mice (48), Fech ${ }^{\text {mIPas } m \text { mPas, }}$ were obtained from Xavier Montagutelli (Institute Pasteur, Paris, France). Mice were backcrossed at least 10 generations on the C57BL/6J background (The Jackson Laboratory). C57BL/6J (CD45.2) and B6-CD45.1 congenic (stock 002014, The Jackson Laboratory) were crossed to generate $\mathrm{CD} 45.1 / 2 \mathrm{~F} 1$ mice as transplant recipients. Adult control Flvcr ${ }^{++} ;$Mxcre (++ Mx) and Flvcrflff; Mxcre (FF Mx) were treated with 3 i.p. injections of $7.5 \mathrm{mg} / \mathrm{kg}$ polyI-polyC (GE Healthcare) 48 hours apart as described (13) to ablate FLVCR1a and FLVCR1b expression. Any Flvcr1-deleted mice with rbc counts below $2 \mathrm{M} / \mu \mathrm{l}$ were euthanized and excluded from subsequent analysis. Donor mice for transduction studies were treated with $3 \mathrm{mg}$ fluorouracil (APP Pharmaceuticals) 11 days after deletion with polyI-polyC. Total marrow was harvested 3 days later and cultured overnight in IMDM with $10 \%$ FBS, $20 \mathrm{ng} / \mathrm{ml}$ murine IL-3, $50 \mathrm{ng} / \mathrm{ml}$ murine SCF, and $100 \mathrm{ng} / \mathrm{ml}$ human IL-6 (PeproTech). Marrow was transduced with bicistronic retroviral vectors expressing GFP in the second position, and either Flvcrla (13), Flvcrlb (14), or Hmox1 (OriGene), in the first position, at a MOI of 5 as described previously (49). Three million transduced cells were transplanted i.v. into each myeloablated (11 Gy) recipient. Mice transplanted with transduced marrow cells were treated with $2 \mathrm{mg} / \mathrm{ml}$ neomycin in drinking water for 3 weeks to prevent opportunistic infections prior to reconstitution of the granulocyte lineage. All mice were maintained under specific pathogen-free conditions with free access to food and water. Iron-deficient diet (TD 99397; <6 ppm iron) was from Harlan Teklad. INH (Sigma-Aldrich) or carrier control (water) 
was given by oral gavage at $50 \mathrm{mg} / \mathrm{kg} 5$ days a week for 8 weeks. Serum EPO concentrations were determined by ELISA (R\&D Systems).

Flow cytometry analysis. Peripheral blood samples were collected from the retro-orbital sinus and analyzed on a HemaVet 950FS (Drew Scientific) for CBC analysis; they were also analyzed by flow cytometry on FacsCanto II or LSR II cytometers, or an Aria III cell sorter (BD Biosciences). Platelets and rbc were analyzed directly after dilution, while rbc lysed samples were used for wbc analysis with CD3, CD4, CD8, B220, GR1, CD45.1, and CD45.2 mAbs (BD Biosciences and eBioscience) to determine the frequency of GFP in each donor cell population. Flow cytometry analysis and sorting of whole BM was performed with B220, Gr1, CD11b, Ter119, CD71, and CD44 mAbs to identify developing erythroblasts. We modified the erythroblast gating protocol of Chen et al. $(20,21)$ to include $\mathrm{B}^{2} 2 \mathrm{O}^{-} \mathrm{Gr1}^{-} \mathrm{CD} 11 \mathrm{~b}^{-}\left(\mathrm{lin}^{-}\right)$ $\mathrm{CD} 1^{+}$Ter119- ${ }^{-}$erythroid progenitors in population I, as described in Figure 1 and Supplemental Figure 2. Endogenous PPIX levels in each population were detected with excitation/detection wavelengths of 405/650 nm. Cytoplasmic ROS were detected with CM-H2DCFCA or CellROX Deep Red, while mitochondrial ROS were detected with MitoSOX Red (Invitrogen). Apoptotic and dead cells were detected with Annexin V and 7AAD (BD Biosciences). Flow cytometry data was analyzed with FlowJo software (Tree Star Inc.).

Real-time PCR. Total RNA was isolated from samples with Trizol (Invitrogen), and $0.5 \mu \mathrm{g}$ of total RNA was used to make cDNA with iScript (Bio-Rad) according to the manufacturer's instructions. Diluted cDNA was detected via quantitative PCR (qPCR) on an iQ5 Real-Time PCR machine (Bio-Rad) using Probe Fast Mastermix (Kapa Biosystems). We evaluated 19 reference genes (Bio-Rad PrimePCR reference gene plate) and found that $\beta$-actin mRNA levels change the least during erythroblast development from proerythroblasts through reticulocytes; thus, normalized expression levels were calculated according to the $\Delta \Delta \mathrm{Ct}$ method (50) using $\beta$-actin as the reference gene and LNPC as the reference sample. Because $\beta$-actin expression decreases during erythroid development (see Figure 2), relative expression levels of genes normalized to $\beta$-actin will be overestimated as $\beta$-actin expression diminishes during erythroid development. To correct this overestimation, we multiplied individual normalized gene expression data by the mean expression level of $\beta$-actin for its respective population. The qPCR probe set for Flvcr1 (13) only detects transcripts containing exons 3 and 4, which are required for functional proteins and are present in both Flvcrla and Flvcrlb. The qPCR probe for Flvcrlb detects all transcripts that contain the common exon 2 and the preceding untranslated region, which is unique to Flvcrlb (14). Probe sets were designed with the RealTime PCR design tool (Integrated DNA Technologies), and all primers are listed in Supplemental Table 5.

Heme content analysis. Total cellular heme levels were detected according to established methods $(51,52)$. Briefly, sorted cells were lysed in RIPA buffer at a concentration of $10^{6}$ cells per ml, diluted 10 -fold in water, and heated to $100^{\circ} \mathrm{C}$ for 30 minutes in 10 -fold excess $2 \mathrm{M}$ oxalic acid. The concentration of heme was determined by measuring the conversion to PPIX via spectrofluorometry using excitation/ emission wavelengths of 400/662 nm (Infinite M200, Tecan).

Statistics. All data are presented as mean \pm SD of the indicated number of biological replicates. Significance testing was performed with the Student's 2-tailed $t$ test, the log-rank (Mantel-Cox) test (survival curve analysis), and 1-way ANOVA with the Tukey-Kramer post hoc test (Prism version 5.03, GraphPad Software). $P \leq 0.05$ was considered significant.

Study approval. All animal studies were reviewed and approved by the University of Washington Institutional Animal Care and Use Committee.

\section{Author contributions}

RTD designed and conducted experiments, collected and analyzed data, and wrote the manuscript. SRP and CS conducted experiments and collected data. MSB conducted experiments, and collected and analyzed data. SBK and JLA designed experiments, analyzed data, and wrote the manuscript.

\section{Acknowledgments}

This work was supported by NIH grant HLO31823 to J.L. Abkowitz. The authors would like to thank Mary Philip for many helpful discussions, Emanuela Tolosano (University of Torino, Torino, Italy) for providing the Flvcr $1 b$ cDNA clone, Nancy Andrews for the transferrin receptor mutant mice, and Xavier Montagutelli for the Ferrochelatase mutant mice. Flow cytometry and cell sorting was performed at the University of Washington Department of Immunology and Department of Pathology flow cytometry core facilities.

Address correspondence to: Janis L. Abkowitz, University of Washington Department of Medicine, Division of Hematology, Box 357710, Seattle, Washington 98195, USA. Phone: 206.685.7877; E-mail:janabk@u.washington.edu.

Marilyn Sanchez-Bonilla's present address is: Karp Family Research Laboratory, Boston Children's Hospital, Boston, Massachusetts, USA.
1. Ferreira GC, Gong J. 5-Aminolevulinate synthase and the first step of heme biosynthesis. J Bioenerg Biomembr. 1995;27(2):151-159.

2. Ajioka RS, Phillips JD, Kushner JP. Biosynthesis of heme in mammals. Biochim Biophys Acta. 2006;1763(7):723-736

3. Gotoh S, Nakamura T, Kataoka T, Taketani S. Egr-1 regulates the transcriptional repression of mouse delta-aminolevulinic acid synthase 1 by heme. Gene. 2011;472(1-2):28-36.

4. Melefors O, Goossen B, Johansson HE, Stripecke R, Gray NK, Hentze MW. Translational control of 5-aminolevulinate synthase mRNA by ironresponsive elements in erythroid cells. J Biol
Chem. 1993;268(8):5974-5978.

5. Dandekar T, et al. Identification of a novel iron-responsive element in murine and human erythroid delta-aminolevulinic acid synthase mRNA. EMBO J. 1991;10(7):1903-1909.

6. Wilkinson N, Pantopoulos K. The IRP/IRE system in vivo: insights from mouse models. Front Pharmacol. 2014;5:176.

7. Tahara T, et al. Heme positively regulates the expression of beta-globin at the locus control region via the transcriptional factor Bach1 in erythroid cells. J Biol Chem. 2004;279(7):5480-5487.

8. Chen JJ. Translational control by heme-regulated eIF2alpha kinase during erythropoiesis. Curr
Opin Hematol. 2014;21(3):172-178.

9. Ponka P, Schulman HM. Regulation of heme synthesis in erythroid cells: hemin inhibits transferrin iron utilization but not protoporphyrin synthesis. Blood. 1985;65(4):850-857.

10. Balla G, Vercellotti GM, Muller-Eberhard U, Eaton J, Jacob HS. Exposure of endothelial cells to free heme potentiates damage mediated by granulocytes and toxic oxygen species. $L a b$ Invest. 1991;64(5):648-655.

11. Garcia-Santos D, et al. Heme oxygenase 1 is expressed in murine erythroid cells where it controls the level of regulatory heme. Blood. 2014;123(14):2269-2277. 
12. Quigley JG, et al. Identification of a human heme exporter that is essential for erythropoiesis. Cell. 2004;118(6):757-766.

13. Keel SB, et al. A heme export protein is required for red blood cell differentiation and iron homeostasis. Science. 2008;319(5864):825-828.

14. Chiabrando $\mathrm{D}$, et al. The mitochondrial heme exporter FLVCR1b mediates erythroid differentiation. J Clin Invest. 2012;122(12):4569-4579.

15. Chiabrando D, Tolosano E. Diamond Blackfan anemia at the crossroad between ribosome biogenesis and heme metabolism. Adv Hematol. 2010;2010:790632.

16. Van Putten LM. The life span of red cells in the rat and the mouse as determined by labeling with DFP32 in vivo. Blood. 1958;13(8):789-794.

17. Von Ehrenstein G. The life span of the erythrocytes of normal and tumour bearing mice as determined by glycine-2-14C. Acta Physiol Scand. 1958;44(1):80-91.

18. Socolovsky M, Nam H-s, Fleming MD, Haase VH, Brugnara C, Lodish HF. Ineffective erythropoiesis

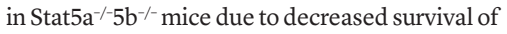
early erythroblasts. Blood. 2001;98(12):3261-3273.

19. Zhang J, Socolovsky M, Gross AW, Lodish HF. Role of Ras signaling in erythroid differentiation of mouse fetal liver cells: functional analysis by a flow cytometry-based novel culture system. Blood. 2003;102(12):3938-3946.

20. Chen K, Liu J, Heck S, Chasis JA, An X, Mohandas $\mathrm{N}$. Resolving the distinct stages in erythroid differentiation based on dynamic changes in membrane protein expression during erythropoiesis. Proc Natl Acad Sci U S A. 2009;106(41):17413-17418.

21. Liu J, et al. Quantitative analysis of murine terminal erythroid differentiation in vivo: novel method to study normal and disordered erythropoiesis. Blood. 2013;121(8):e43-e49.

22. Terszowski G, et al. Prospective isolation and global gene expression analysis of the erythrocyte colony-forming unit (CFU-E). Blood. 2005;105(5):1937-1945.

23. Flygare J, Rayon Estrada V, Shin C, Gupta S, Lodish HF. HIF1alpha synergizes with glucocorticoids to promote BFU-E progenitor selfrenewal. Blood. 2011;117(12):3435-3444.

24. $\mathrm{Li} \mathrm{J}$, et al. Isolation and transcriptome analyses of human erythroid progenitors: BFU-E and CFU-E. Blood. 2014;124(24):3636-3645.

25. Testa NG, Onions D, Jarrett O, Frassoni F, Eliason JF. Haemopoietic colony formation (BFU-E,
GM-CFC) during the development of pure red cell hypoplasia induced in the cat by feline leukaemia virus. Leuk Res. 1983;7(2):103-116.

26. Abkowitz JL. Retrovirus-induced feline pure red cell aplasia: pathogenesis and response to suramin. Blood. 1991;77(7):1442-1451.

27. Yanatori I, Yasui Y, Miura K, Kishi F. Mutations of FLVCR1 in posterior column ataxia and retinitis pigmentosa result in the loss of heme export activity. Blood Cells Mol Dis. 2012;49(1):60-66.

28. Yang $Z$, et al. Kinetics and specificity of feline leukemia virus subgroup C receptor (FLVCR) export function and its dependence on hemopexin. J Biol Chem. 2010;285(37):28874-28882.

29. Ponka P. Tissue-specific regulation of iron metabolism and heme synthesis: distinct control mechanisms in erythroid cells. Blood. 1997;89(1):1-25.

30. Byon JC, Chen J, Doty RT, Abkowitz JL. FLVCR is necessary for erythroid maturation, may contribute to platelet maturation, but is dispensable for normal hematopoietic stem cell function. Blood. 2013;122(16):2903-2910.

31. Lyoumi S, et al. Increased plasma transferrin, altered body iron distribution, and microcytic hypochromic anemia in ferrochelatase-deficient mice. Blood. 2007;109(2):811-818.

32. Levy JE, Jin O, Fujiwara Y, Kuo F, Andrews NC. Transferrin receptor is necessary for development of erythrocytes and the nervous system. Nat Genet. 1999;21(4):396-399.

33. Keel SB, et al. Evidence that the expression of transferrin receptor 1 on erythroid marrow cells mediates hepcidin suppression in the liver. Exp Hematol. 2015;43(6):469-478.

34. Ponka P, Neuwirt J. Regulation of iron entry into reticulocytes. I. Feedback inhibitory effect of heme on iron entry into reticulocytes and on heme synthesis. Blood. 1969;33(5):690-707.

35. Dixon SJ, et al. Ferroptosis: an iron-dependent form of nonapoptotic cell death. Cell. 2012;149(5):1060-1072.

36. Dixon SJ, Stockwell BR. The role of iron and reactive oxygen species in cell death. Nat Chem Biol. 2014;10(1):9-17.

37. Jiang $\mathrm{L}$, et al. Ferroptosis as a p53-mediated activity during tumour suppression. Nature. 2015;520(7545):57-62.

38. Poss KD, Tonegawa S. Heme oxygenase 1 is required for mammalian iron reutilization. Proc Natl Acad Sci U S A. 1997;94(20):10919-10924.

39. Cianetti L, et al. Expression of alternative transcripts of ferroportin-1 during human erythroid differentiation. Haematologica. 2005;90(12):1595-1606.

40. Zhang DL, Hughes RM, Ollivierre-Wilson H, Ghosh MC, Rouault TA. A ferroportin transcript that lacks an iron-responsive element enables duodenal and erythroid precursor cells to evade translational repression. Cell Metab. 2009;9(5):461-473.

41. Zhu BM, et al. Hematopoietic-specific Stat5-null mice display microcytic hypochromic anemia associated with reduced transferrin receptor gene expression. Blood. 2008;112(5):2071-2080

42. Doherty L, et al. Ribosomal protein genes RPS10 and RPS26 are commonly mutated in Diamond-Blackfan anemia. Am J Hum Genet. 2010;86(2):222-228.

43. Ebert BL, et al. Identification of RPS14 as a 5qsyndrome gene by RNA interference screen. Nature. 2008;451(7176):335-339.

44. Li H, Ginzburg YZ. Crosstalk between iron metabolism and erythropoiesis. Adv Hematol. 2010;2010:605435.

45. Gardenghi S, et al. Hepcidin as a therapeutic tool to limit iron overload and improve anemia in $\beta$-thalassemic mice. J Clin Invest. 2010;120(12):4466-4477.

46. Guo $S$, et al. Reducing TMPRSS6 ameliorates hemochromatosis and $\beta$-thalassemia in mice. JClin Invest. 2013;123(4):1531-1541.

47. Rivella $S$. The role of ineffective erythropoiesis in non-transfusion-dependent thalassemia. Blood Rev. 2012;26(suppl 1):S12-S15.

48. Boulechfar S, et al. Ferrochelatase structural mutant (Fechm1Pas) in the house mouse. Genomics. 1993;16(3):645-648.

49. Doty RT, Sabo KM, Chen J, Miller AD, Abkowitz JL. An all-feline retroviral packaging system for transduction of human cells. Hum Gene Ther. 2010;21(8):1019-1027.

50. Pfaffl MW. A new mathematical model for relative quantification in real-time RT-PCR. Nucleic Acids Res. 2001;29(9):e45.

51. Sassa S. Sequential induction of heme pathway enzymes during erythroid differentiation of mouse Friend leukemia virus-infected cells. J Exp Med.1976;143(2):305-315.

52. Nie G, Chen G, Sheftel AD, Pantopoulos K, Ponka $P$. In vivo tumor growth is inhibited by cytosolic iron deprivation caused by the expression of mitochondrial ferritin. Blood. 2006;108(7):2428-2434. 